The Milky Way Unravelled by Gaia: GREAT Science from the Gaia Data Releases N.A. Walton, F. Figueras, L. Balaguer-Núñez and C. Soubiran (eds)

EAS Publications Series, 67-68 (2014) 1-3

\title{
PREFACE: THE MILKY WAY UNRAVELLED BY GAIA: GREAT SCIENCE FROM THE GAIA DATA RELEASES
}

\author{
N.A. Walton ${ }^{1}$, F. Figueras ${ }^{2}$, L. Balaguer-Núnez ${ }^{2}$ and C. Soubiran ${ }^{3}$
}

Abstract. This paper introduces the The Milky Way Unravelled by Gaia conference proceedings.

\section{Gaia and GREAT}

Gaia $^{4}$ is the European Space Agency's cornerstone mission set to revolutionise our understanding of the Milky Way. It successfully launched in December 2013, and is now in its routine operations phase. Its first data is already being issued to the community, these being the Gaia Photometric Science Alerts. The first main Gaia data release will occur in 2016, see http://www. cosmos . esa.int/web/gaia/ gaia-data for details.

The Gaia Research for European Astronomy Training (GREAT) network (Walton et al. 2015), in partnership with ESA, has provided vital scientific networking over the period 2009-2015, preparing a large community of scientists and young researchers to maximise the scientific exploitation of Gaia. In the coming years, the promise of Gaia will start to be realised with the release of increasingly rich Gaia data sets. One element of the GREAT programme has been the GREAT Initial Training Network (GREAT-ITN) ${ }^{5}$. This network involved over thirteen core research institutes, with twenty two partner groups from academia and industry. Over its four years (March 2011-February 2015) it provided training for a cohort of seventeen early stage researchers, all undertaking doctoral research programmes across the GREAT-ITN core institutes, in topics related to the science of Gaia.

\footnotetext{
${ }^{1}$ Institute of Astronomy, University of Cambridge, Madingley Road, Cambridge CB3 0HA, UK; e-mail: naw@ast.cam.ac.uk

2 Departament d'Astronomia i Meteorologia and IEEC-ICC-UB, Universitat de Barcelona, Martí i Franquès 1, 08028 Barcelona Spain

3 Université Bordeaux 1, CNRS, Laboratoire d'Astrophysique de Bordeaux UMR 5804, BP. 89, 33270 Floirac, France

${ }^{4}$ See the Gaia webpage at http://sci.esa.int/gaia/

${ }^{5}$ See the web page at http://www.great-itn.eu for details
} 


\section{The Milky Way unravelled by Gaia conference}

This conference represents the closing meeting of the GREAT-ITN network, but also the first opportunity to present the initial results from Gaia. The conference brought together some one hundred and forty participants from sixteen countries.

The conference website can be found at http://greatconf14.ub.edu, this contains the programme, and the presentation slides given at the conference. There was an active commentary of the meeting over social media - this at the twitter hashtag \#greatconf14. Some images can be found at https://twitter.com/ search?q=\%23greatconf $14 \&$ src=hash\&vertical=def ault\&f=images.

The programme was organised around the main science themes of the GREATITN network:

- Session 1: The GREAT ITN and Gaia: Context and Status

- Session 2: The Physics and Science Promise of Gaia

- Session 3: Planetary Systems: Worlds Near and Far

- Session 4: The Stellar Constituents of the Milky Way

- Session 4A: Star Cluster Evolution

- Session 4B: Stellar Astrophysics

- Session 5: The Origin and History of the Milky Way

- Session 5A: Archaeology of the Milky Way

- Session 5B: Galactic Structure and Evolution

- Session 5C: Gaia, Galactic Surveys, Modelling, Synergies

- Session 5D: Galactic Dynamics

- Session 6: Grand Challenges from Gaia

- Session 6A: The Distance Scale

- Session 6B: The Transient Sky

- Session 7: Beyond Gaia, Beyond GREAT.

Each session included a range of invited presentations, presentations from the GREAT-ITN early stage researchers (ESR), and contributed presentations. There were also a number of poster presentations on display for the week of the conference.

In addition to the main science sessions there were two extended question and answer sessions. These were organised by the GREAT-ITN ESR students. They decided on the questions, and invited inspiring speakers to address each topic. There then followed a lively discussion session involving the audience and the panel composed of the four speakers.

- $\mathrm{Q}+\mathrm{A}$ session: Understanding the formation of the Milky Way in the era of Gaia

- $\mathrm{Q}+\mathrm{A}$ session: What drives the evolution of the Milky Ways disk?

- Q+A session: Scientific synergies of Gaia with ground based spectroscopic

- Q+A session: Exploring Milky Way structure and dynamics. 
This volume provides a written record of the papers and posters presented at the conference, and represents a significant milestone in the era of Gaia.

\section{Reference}

Walton, N.A., Bailer-Jones, C., Brown, A.G.A., et al., 2015, these proceedings 